\title{
油酸钠控制硫酸钙晶须晶面生长的机理研究
}

\author{
郝海青, 袁致涛, 李丽匣, 张 晨 \\ (东北大学资源与上木工程学院, 沈阳 110004)
}

摘 要: 结合油酸钠溶液化学计算、硫酸钲晶须晶面衍射和分子动力学模拟, 研究了硫酸钙晶须不同晶面的结构、 油酸钠在不同晶面的吸附和硫酸钙晶须的晶面生长。结果表明: 硫酸钻晶须晶面中, 钻原子与氧原子、氢原子规律 分布在(200)晶面和(400)晶面表面; 与其它晶面相比, 油酸钠与(200)晶面和(400)晶面的作用能较小, 吸附构型稳定; (200)晶面和(400)晶面上油酸根与䥻离子之间的距离分别为 $0.239 \mathrm{~nm}$ 和 $0.237 \mathrm{~nm}$, 小于作用后的油酸根与钠离子之 间的距离, 得出钻原子是油酸根在晶须晶面作用的活性位点。油酸钠的选择性吸附阻止了(200)晶面和(400)晶面的 生长, 晶面生长速率的差异使得晶须沿 $c$ 轴择优生长。

关 键 词: 硫酸钻晶须; 晶面; 分子动力学; 径向分布函数

中图分类号: TQ174 文献标识码: A

\section{Mechanism of Faces Growth in Preparing Calcium Sulphate Whiskers Using Sodium Oleate}

\author{
HAO Hai-Qing, YUAN Zhi-Tao, LI Li-Xia, ZHANG Chen
}

(School of Resources \& Civil Engineering, Northeastern University, Shenyang 110004, China)

\begin{abstract}
The crystal structure of calcium sulphate whiskers and adsorption of sodium oleate on crystal faces were investigated through methods of solution chemistry calculation, X-ray diffraction analysis and molecular dynamics simulation. Furthermore, the growth of crystal faces was explored in presence of sodium oleate. Results showed that calcium, oxygen and hydrogen atoms were primarily distributed on crystal faces of (200) and (400). Interaction energies between sodium oleate and faces of (200) and (400) were much smaller than those of other faces. Distances between oleic acid and calcium on crystal faces of (200) and (400) were $0.269 \mathrm{~nm}$ and $0.237 \mathrm{~nm}$, respectively, which were smaller than those between oleic acid and sodium. It could reasonably deduce that calcium was the active site of crystal faces. The selective adsorption of sodium oleate prevented growths of (200) face and (400) face. Differences in growth rate of crystal faces made whiskers grow along $c$-axis.
\end{abstract}

Key words: calcium sulphate whiskers; crystal face; molecular dynamics simulation; radial distribution function

硫酸钙晶须是在特定条件下、以单晶形式生长 的具有完整外形和完善内部结构的纤维状单晶 ${ }^{[1]}$ 。 作为新型功能材料, 硫酸钙晶须以其较低的成本、 较高的抗拉强度和弹性模量等优点, 广泛应用于建
筑材料、密封材料、摩擦材料、保温及阻燃材料等, 有着极为广阔的市场发展前景 ${ }^{[2-5]}$ 。晶须的结晶特性 决定晶须的形貌，从而影响其机械强度等性质以及 其在材料领域的应用，通过控制晶须的定向生长可

收稿日期：2016-03-26; 收到修改稿日期：2016-05-04

基金项目：国家自然科学基金(51574061；50502011); 中央高校基本科研业务费专项(N150106004) National Natural Science Foundation of China (51574061; 50502011); Fundamental Research Funds for the Central Universities(N150106004)

作者简介: 郝海青(1990-), 女，博士研究生. E-mail: yesterday1019@163.com

通讯作者: 袁致涛, 教授. E-mail: yuanzhitao@mail.neu.edu.cn 
以得到不同形貌、不同性质的硫酸钙晶须 ${ }^{[6]}$ 。

影响溶液中晶须定向生长的因素有溶液 $\mathrm{pH}$ 、反 应温度、浓度和不同添加剂的使用等 ${ }^{[7]}$ 。其中, 添加 剂能够改变晶须的结晶习性和生长方式, 进而改变 晶须的形貌，且不同添加剂的作用机理不同 ${ }^{[8]}$ 。硫酸 钙晶须制备过程中常用的添加剂可分为无机添加剂 (金属离子等)和有机添加剂(表面活性剂等 $)^{[9-10]}$, 但 这些添加剂的作用机理并不完全清楚。就采用有机 添加剂生长硫酸钻晶须来说, 虽然有研究认为, 不 同溶液环境中有机添加剂对晶体成核及生长的作用 方式可归结为, 有机酸根离子与晶体表面钙原子的 结合 ${ }^{[11]}$ 。但这个观点由于受检测技术的局限而无法 得到证实, 其晶面结构差异与添加剂相互作用的机 理仍有待新方法予以揭示。

近年来, 随着计算机模拟技术的发展, 晶体成 核以及晶体生长的分子模拟逐步成为揭示晶须晶面 生长机理的有效方法 ${ }^{[12-13]}$ 。Yini 等 ${ }^{[14]}$ 和毛修龙等 ${ }^{[15]}$ 分别采用分子模拟法研究了不同添加剂在硫酸沙丁 胺醇和二水硫酸钙晶体不同晶面的吸附行为, 并篎 选了利于特定形貌晶体生长的添加剂, 利用添加剂 与晶须不同晶面的分子动力学模拟, 从微观角度诠 释了添加剂的作用机理, 量化了两者间的作用能, 直观揭示了添加剂对不同晶面的作用过程。杨娜等 ${ }^{[8]}$ 在研究不同添加剂对硫酸钙晶须生长的影响时, 发 现油酸钠对硫酸钙晶须的生长具有较好的促进作 用。袁致涛等 ${ }^{[16]}$ 也发现油酸钠对硫酸钲晶须具有较 好的稳定作用。但两项研究均未能揭示油酸钠调控 硫酸钙晶须不同晶面生长的机理。

本研究基于分子动力学模拟油酸钠在半水硫酸 钲晶须不同晶面的作用能, 分析油酸钠作用前后分 子的变化, 探索了油酸钠对半水硫酸钙晶须形貌的 作用机理, 为添加剂的选择和晶须的可控生长提供
了理论依据。

\section{1 实验方法}

\section{1 实验原料}

实验所用硫酸钙原料 $\left(\omega_{\mathrm{CaSO} 4} \geqslant 92 \%\right)$ 中含有少量 的铝、硅和铁杂质，硫酸钻原料粒度分布不均匀, $d_{90}$ 为 $0.094 \mathrm{~mm}, d_{10}$ 为 $0.009 \mathrm{~mm}$, 其中粒径在 $74 \mu \mathrm{m}$ 左 右的颗粒占 $38.97 \%$, 其粒度组成如表 1 所示。

本研究选用油酸钠 $\left(\mathrm{C}_{18} \mathrm{H}_{33} \mathrm{NaO}_{2}\right.$, 国药集团化学 试剂有限公司, 化学纯)作为制备硫酸钻晶须的添 加剂; 选用无水乙醇 $\left(\mathrm{CH}_{3} \mathrm{CH}_{2} \mathrm{OH}\right.$, 天津市科密欧化 学试剂有限公司, 分析纯)作为分散介质。

\section{2 硫酸钙晶须的制备}

将实验原料配制成质量浓度为 3\%的料浆, 加入油 酸钠后调节初始 $\mathrm{pH}$ 至 9.25 , 搅拌 $30 \mathrm{~min}$ 后加入反应釜 (FYF-10, 无锡恒金金石化装备厂)进行水热合成反应，在 $120^{\circ} \mathrm{C}$ 条件下反应 $2 \mathrm{~h}$ 。反应结束后, 释放釜内气体, 待 釜内气压降到大气压强时, 取出物料, 进行快速脱水、 烘干, 最终采用扫描电子显微镜(SSX-550, 日本岛津 公司)观察硫酸钙晶须产物形貌。

\section{3 硫酸钙晶须的性能表征}

对烘干后的水热产品进行 $\mathrm{X}$ 射线衍射分析 (X'Pert Pro, 荷兰帕纳科公司), 并依据得到的图谱 对水热产品进行晶面衍射强度分析。 $\mathrm{X}$ 射线衍射仪 扫描范围为 $2 \theta=5^{\circ} \sim 90^{\circ}$, 步长 $0.033^{\circ}$, 每步停留时间 $20.68 \mathrm{~s}, \mathrm{Cu}$ 靶辐射, 入射线波长 $0.1541 \mathrm{~nm}$, 扫描时 间 $7 \mathrm{~min}$, 工作温度 $25^{\circ} \mathrm{C}$ 。

\section{4 分子动力学模拟}

采用分子动力学模拟油酸钠在硫酸钙晶须不同 晶面的吸附, 首先需要构建晶面, 并对不同晶面模 型进行几何优化, 优化过程中固定底层原子, 建立 超晶胞; 其次对药剂分子进行几何优化, 将优化好

表 1 试样粒度分布统计表

Table1 Statistical data of sample size distribution

\begin{tabular}{ccccc}
\hline \multirow{2}{*}{ Size fraction/mm } & \multicolumn{3}{c}{ Productivity $/ \%$} & Cumulative productivity /\% \\
\cline { 2 - 4 } & Sample A & Sample B & Sample C & 19.76 \\
\hline 0.074 & 20.35 & 19.17 & 19.76 & 58.73 \\
$0.038 \sim 0.074$ & 39.39 & 38.54 & 38.97 & 73.01 \\
$0.027 \sim 0.038$ & 14.27 & 14.29 & 14.28 & 81.10 \\
$0.019 \sim 0.027$ & 7.92 & 8.25 & 8.09 & 85.00 \\
$0.014 \sim 0.019$ & 3.67 & 4.12 & 3.90 & 88.60 \\
$010 \sim 0.014$ & 3.58 & 3.63 & 11.40 & 100.00 \\
\hline
\end{tabular}


的初始构型, 几何优化寻找最佳吸附平衡位置后进 行动力学模拟, 使构型能量收玫。动力学模拟输出 优化的轨迹结构, 吸附最优模型是能量最低的构型, 对最优模型进行相互作用能计算 ${ }^{[17]}$ 。

油酸钠与硫酸钲晶须不同晶面作用的分子模拟 采用 Materials Studio 7.0 中 Forcite Plus 模块。分子 构型优化采用 Smart 算法, 收玫精度为 Ultra-fine, 力场为 Dreiding 力场, 电荷分配采用 $\mathrm{QEq}$ charges, 范德华作用采用 Group based, 静电作用均采用 Ewald 求和法, 计算精度为 $0.001 \mathrm{kcal} / \mathrm{mol}^{[18-21]}$ 。

系综表示具有相同条件系统的集合，可分为正 则系综(NVT)、等温定压系综(NPT)等, 正则系统 $(N V T)$ 是具有相同的分子数 $N$ 、体积 $V$ 和温度 $\mathrm{T}$ 的 系统的集合 ${ }^{[22]}$ 。药剂分子作用模型在 $298 \mathrm{~K}$ 温度条 件下采用 NVT 系综进行动力学模拟至收玫后, 温 度设为 $393 \mathrm{~K}$, 采用 NVT 系综, 时间步长设为 $1 \mathrm{fs}$, 总模拟时间为 $50 \mathrm{ps}$ 。

\section{2 结果与分析}

\section{1 油酸钠的溶液化学计算}

图 1 是油酸钠用量为 $0.15 \%\left(\mathrm{C}_{\mathrm{T}}=1.64 \times 10^{-4} \mathrm{~mol} / \mathrm{L}\right)$ 时得到的硫酸钙晶须 SEM 照片, 晶须呈针状、长柱 状, 长度可达 $112 \mu \mathrm{m}$, 长径比为 40 。

由图 2 可知, 当水热合成反应的 $\mathrm{pH}$ 调至 9.25 时, 溶液中起主要作用的成分是油酸根 $\left(\mathrm{RCOO}^{-}\right)$, 油酸根可以与硫酸钙晶须晶面上活性位点钙离子发 生化学反应, 生成稳定化合物油酸钙。油酸钙的溶 度积 $\left(1.0 \times 10^{-15.4}\right)$ 远小于硫酸钙的溶度积 $\left(9.1 \times 10^{-6}\right)$, 溶液中易形成油酸钲稳定化合物吸附在硫酸钙晶须 的晶面, 进而阻止了此晶面的生长 ${ }^{[23]}$ 。硫酸钙晶须 不同的晶面有不同结构, 表面的原子分布也存在差

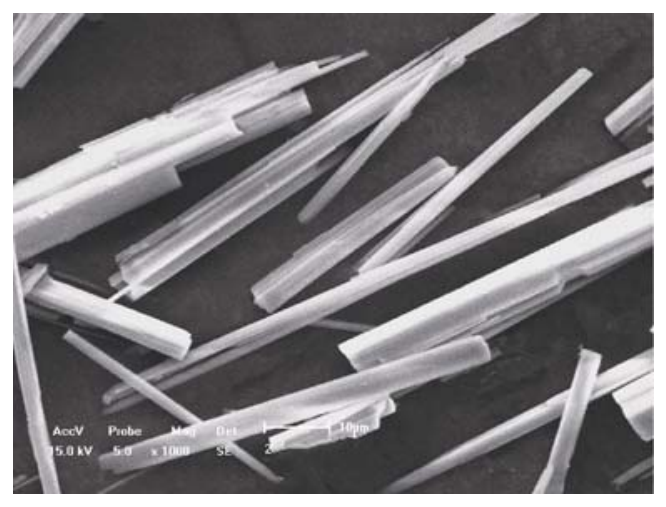

图 $10.15 \%$ 油酸钠作用下硫酸钙晶须 SEM 形貌

Fig. 1 SEM image of calcium sulfate whisker with $0.15 \%$ sodium oleate

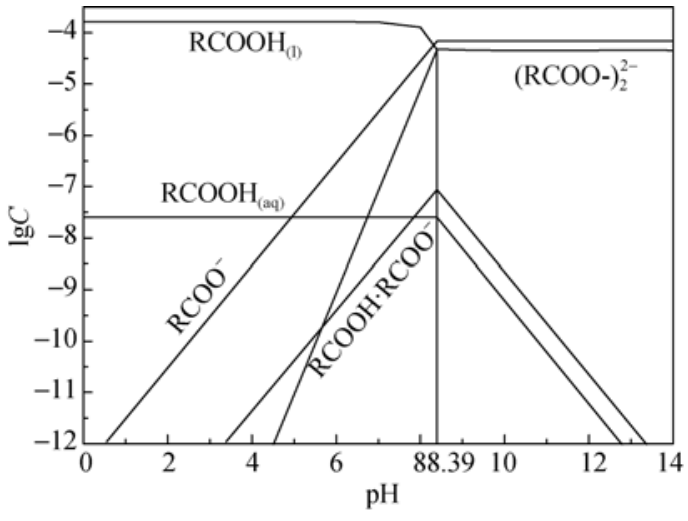

图 2 油酸钠溶液各组分的 $\log \mathrm{C}-\mathrm{pH}$ 图 $\left(C_{\mathrm{T}}=1.64 \times 10^{-4} \mathrm{~mol} / \mathrm{L}\right)$ Fig. 2 The $\log \mathrm{C}-\mathrm{pH}$ relationship of sodium oleate solution system $\left(C_{\mathrm{T}}=1.64 \times 10^{-4} \mathrm{~mol} / \mathrm{L}\right)$

异, 油酸根对于晶面的吸附具有选择性, 造成了各 晶面生长速度的差异, 硫酸钻晶须因其轴向生长速 度大于径向生长速度而呈现长柱状和针状。

\section{2 硫酸钻晶须晶面衍射分析}

图 3 表明，以油酸钠为添加剂水热合成的硫酸 钙晶须, (400)晶面和(200)晶面对应的衍射峰均较 强。(310)晶面的衍射峰强度比(530)晶面强, 为第三 强峰。(400)、(200)、(310)、(530)和(510)晶面均为 平行于 $c$ 轴的晶面, 由此可以得出硫酸钲晶须沿 $c$ 轴生长的速度远大于垂直 $c$ 轴生长的速度, 即油酸 钠促进了晶须沿 $c$ 轴择优生长。

\section{3 硫酸钙晶须晶面分析}

不同晶面有着不同的表面结构，与油酸钠作用 强度大小不同。晶面活性位点多, 油酸钠吸附作用强, 油酸钠的吸附使得表面能降低，且溶液中硫酸根离 子和钙离子的定向吸附受到抑制, 晶面的相对生长 速率降低, 此晶面生长受到抑制, 晶面显现; 晶面活 性位点少, 油酸钠吸附作用弱, 此晶面具有一定的

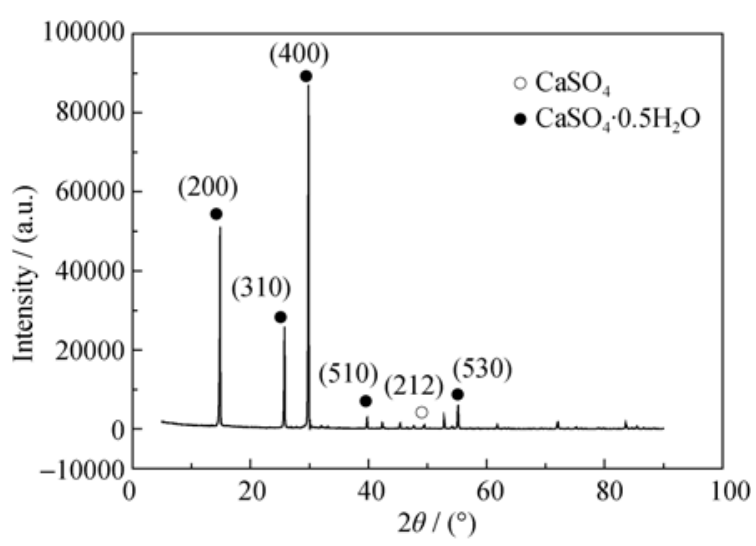

图 $30.15 \%$ 油酸钠作用下硫酸钲晶须晶面衍射强度

Fig. 3 Diffraction strength of crystal faces with $0.15 \%$ sodium oleate 
生长速度, 晶面生长成其他参数晶面, 此晶面逐渐 缩小甚至消失 ${ }^{[24-25]}$ 。选取上述 X 射线衍射图谱中部 分晶面和参考晶面进行建模，建模晶面包括: (200)、 (400)、(310)、(530)、(510)和(002)晶面，其中(002) 晶面属于参考晶面 ${ }^{[15]}$ 。

图 4 所示的晶面结构中, (400)晶面和(200)晶 面具有相似的表面原子分布, 裸露的钙原子、氧 原子和氢原子有规律地分布在表面，使得油酸钠 在这类晶面的吸附作用相近, 硫酸钙晶须中 (400) 晶面和(200)晶面显现。(310)晶面表面氧原子和氢 原子交替排列，没有与油酸根反应的活性位点， (310) 晶面在溶液过饱和作用下继续生长。(530)晶 面和(510)晶面分布的是硫原子、氢原子、氧原子 和少数钻原子, 油酸钠吸附作用弱, 晶面具有一 定的生长速度 ${ }^{[25]}$ 。

(002)晶面为垂直于 $c$ 轴的径向晶面，与(200)晶 面和(400)晶面有着相似的表面原子组成, 但与(200) 晶面和(400)晶面不同的是：(002)晶面少了单独排列 的水分子, 钙原子在表面的分布率大, 空间位阻的 存在不利于油酸根的吸附 ${ }^{[26]}$ 。少数径向晶面占硫酸 钲晶须总表面的比例小, 因而图 3 中未检测到径向 晶面。

\section{4 分子动力学模拟}

原子通过化学键的作用形成分子，分子之间则 通过非键作用(即分子间作用力)形成分子聚集体, 分子间作用力决定了分子的堆积方式和排列方式, 从而决定了分子的稳定性、界面性质等 ${ }^{[24]}$ 。分子动 力学模拟可以实现在分子水平上研究油酸钠与不同 晶面的作用能，从分子角度解释油酸钠的作用差异， 最终实现控制硫酸钙晶须的定向生长。

相互作用能 $(\Delta E)$ 表示作用体系的稳定性，相互 作用能越负，作用体系稳定性越高，油酸钠越容易 作用在晶面上，相互作用能为零或正值，说明油酸 钠不吸附在晶面上 ${ }^{[27]}$ 。表 2 所示油酸钠在晶面的作 用能均为负值, 说明油酸钠与轴向晶面和径向晶 面(002)均作用，其作用能大小顺序为： $\Delta E_{(200)}<$ $\Delta E_{(002)}<\Delta E_{(400)}<\Delta E_{(310)}<\Delta E_{(530)}<\Delta E_{(510)}$, 基本与晶 面衍射强度结果一致。油酸钠在 $(200)$ 晶面作用最 强，抑制了(200)晶面生长，(200)晶面保留，晶面衍 射强度较高。油酸钠在(400)晶面的作用较弱于(200) 晶面，与(200)晶面类似。除此之外，平行于 $c$ 轴生 长的(310)晶面、(530)晶面, (510)晶面均与油酸钠作 用, 晶面保留。垂直于 $c$ 轴生长的(002)晶面与油酸 钠作用较强，晶面保留，由于轴向晶面面积总和远
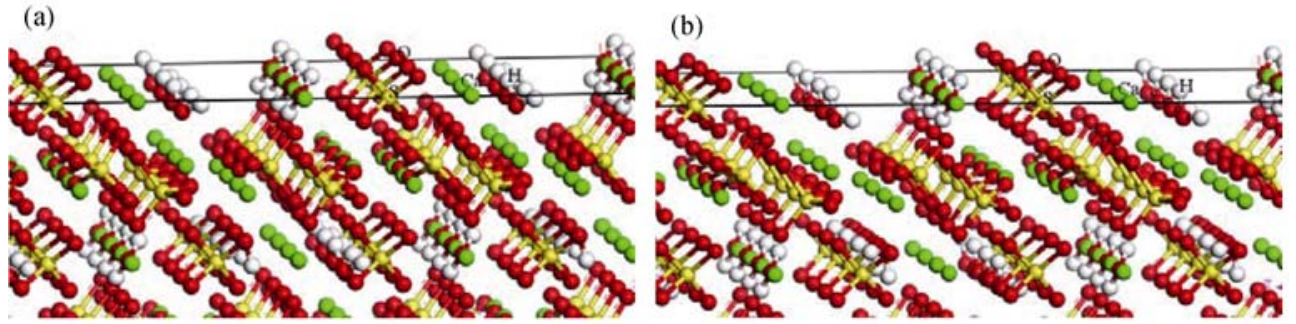

(c)

(d)
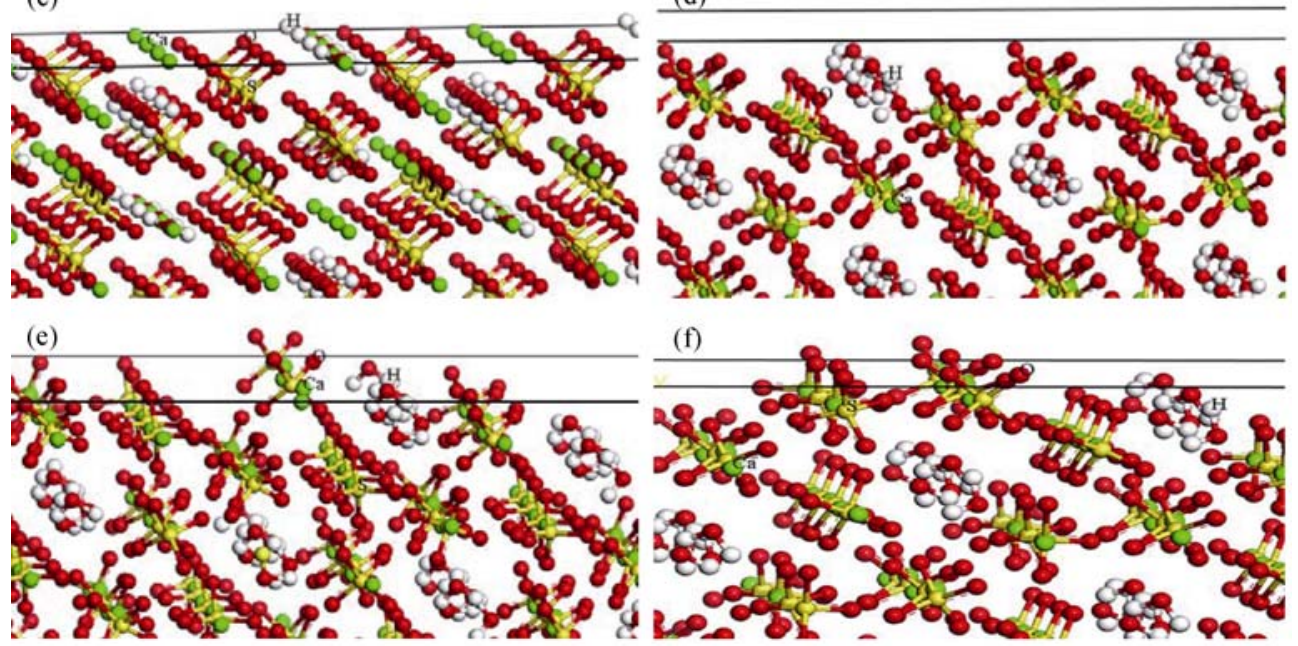

图 4 晶面结构图

Fig. 4 Structure of some crystal faces

(a) (200) face; (b) (400) face; (c) (002) face; (d) (310) face; (e) (530) face; (f) (510) face (Note: Crystal faces are defined by two black lines which are parallel to each other) 
表 2 油酸钠在不同晶面的作用能

Table 2 Interaction energy of sodium oleate on different faces

\begin{tabular}{ccccccc}
\hline \multirow{2}{*}{ additive } & \multicolumn{5}{c}{ Interaction energy, $\Delta E /\left(\mathrm{kJ} \cdot \mathrm{mol}^{-1}\right)$} \\
\cline { 2 - 6 } & $(200)$ & $(400)$ & $(002)$ & $(310)$ & $(530)$ & $(510)$ \\
\hline Sodium oleate & -3259.43 & -2827.17 & -3003.67 & -1051.77 & -913.50 & -453.43 \\
\hline
\end{tabular}

远大于径向晶面面积, $\mathrm{X}$ 射线衍射未能检测到径向 晶面的衍射峰。

\section{5 径向分布函数}

径向分布函数 $g(\mathrm{r})$ 表示某粒子在指定粒子周围 的空间分布率，第一个峰出现的位置可用来分析原 子之间的相互作用与否, 其表达式如式(1)所示 ${ }^{[28]}$ 。

$$
\mathrm{g}_{\mathrm{AB}}(r)=\frac{1}{x_{\mathrm{A}} x_{\mathrm{B}} \rho N}\left\langle\sum_{i=1}^{N_{\mathrm{A}}} \sum_{i=1}^{N_{\mathrm{B}}} \delta\left(r-r_{i}+r_{i}\right)\right\rangle
$$

式中, $x_{\mathrm{A}}, x_{\mathrm{B}}-\mathrm{A} 、 \mathrm{~B}$ 元素的摩尔分数;

$N_{\mathrm{A}}, N_{\mathrm{B}}-\mathrm{A} 、 \mathrm{~B}$ 元素的原子个数;

$N$-总原子数;

$\rho$-数密度;

$\delta(x)$-位置函数。

对油酸钠在硫酸钙晶须晶面作用的分子动力学 模拟结果进行分析, 得到作用前、后油酸根离子与 钠离子之间的距离, 并得到油酸根离子 $\mathrm{RCOO}^{-}$与晶 面上钙离子的径向分布函数。

对比表 3 和图 5 相应数据可得, (200)晶面和(400) 晶面的油酸根与钠离子之间的距离分别为 $0.467 \mathrm{~nm}$ 和 $0.495 \mathrm{~nm}$, 大于作用前油酸钠中油酸根与钠离子 之间距离 $0.268 \mathrm{~nm}$, 且油酸根与钙离子之间距离 $0.239 \mathrm{~nm}$ 和 $0.237 \mathrm{~nm}$ 与作用前油酸钠中油酸根与钠 离子之间距离相近, 说明几乎所有油酸根与晶面上 的钙离子发生作用, 生成油酸钻吸附在晶面上 ${ }^{[29]}$ 。 (310)晶面、(530)晶面、(510)晶面的油酸根与钠离子 之间的距离与作用前相近, 晶面上油酸根与钙离子 之间距离在 $0.250 \sim 0.260 \mathrm{~nm}$ 之间, 即油酸根与钠离 子和钙离子均有作用, 所以部分油酸根吸附在晶面 上。(002)晶面油酸根与钙离子之间距离 $0.239 \mathrm{~nm}$ 小 于油酸根与钠离子之间的距离 $0.433 \mathrm{~nm}$, 即油酸根 与晶面上铂离子反应生成油酸钙吸附在(002)表面。 图 5 中, (002)晶面粒子径向分布函数的第二峰出现 在 $r=0.471 \mathrm{~nm}$ 处, (200)晶面和(400)晶面的第二峰 出现位置分别为 $r=0.281 \mathrm{~nm}$ 和 $r=0.277 \mathrm{~nm}$ 处,
而(002)晶面与(200)、(400)晶面有相似的表面结构, 可知油酸根在(002)晶面的吸附量小于在(200)晶面 和(400)晶面的吸附量 ${ }^{[29]}$, 部分油酸根在(002)晶面 的吸附造成钙原子与溶液中的硫酸根结合, 因而 (002)晶面具有一定的生长速度, 此为 $\mathrm{X}$ 射线衍射检 测不到(002)晶面衍射峰的主要原因。因此, 油酸钠 对上述晶面的生长均产生作用, 其作用程度大小与 晶面组成相关。

\section{3 结论}

结合 $\mathrm{X}$ 射线衍射结果，对模拟作用能和径向分 布函数进行分析, 得出硫酸钙晶须晶面生长差异性 的缘由, 结论如下:

1) 硫酸钙晶须晶面衍射强度分析得知晶须沿 $c$ 轴生长, 保留了平行于 $c$ 轴的(400)、(200)等晶面;

2) 硫酸䥻晶须不同的晶面结构, 决定了不同 晶面与油酸钙作用强度的大小, 平行于 $c$ 轴的晶面 表面均分布有钙离子，含有裸露钙离子的表面与油 酸钙作用强，此晶面生长受到抑制;

3) 油酸钠与晶面相互作用能和径向分布函数 结果表明, 油酸钠选择性地作用于不同晶面, 油 酸钙的生成改变了油酸根与晶面原子和钠离子之 间的距离, (200)晶面和(400)晶面的油酸根与钠离 子之间的距离大于油酸根与钙离子之间距离, 即 油酸根与晶面上的钙离子发生作用, 生成油酸钙 吸附在晶面上。(310)晶面、(530)晶面、(510)晶面 的油酸根与钠离子之间的距离与作用前相近, 即 油酸根与钠离子和钙离子均有作用, 部分油酸根 吸附在晶面上。分子模拟结果与晶面衍射结果基 本一致;

4) 从分子层面探究油酸钠对硫酸䥻晶须晶面 生长的影响, 得出微观作用机理, 可为选择晶型控 制剂提供理论依据。

表 3 不同晶面上油酸根与钠离子的距离

Table 3 Distance between oleic acid and sodium on different faces

\begin{tabular}{cccccccc}
\hline Distance, $r / \mathrm{nm}$ & Before adsorption & $(200)$ & $(400)$ & $(310)$ & $(530)$ & $(510)$ & $(002)$ \\
\hline$r_{\mathrm{COO}-\mathrm{Na}}{ }^{+}$ & 0.268 & 0.467 & 0.495 & 0.253 & 0.325 & 0.267 & 0.433 \\
\hline
\end{tabular}



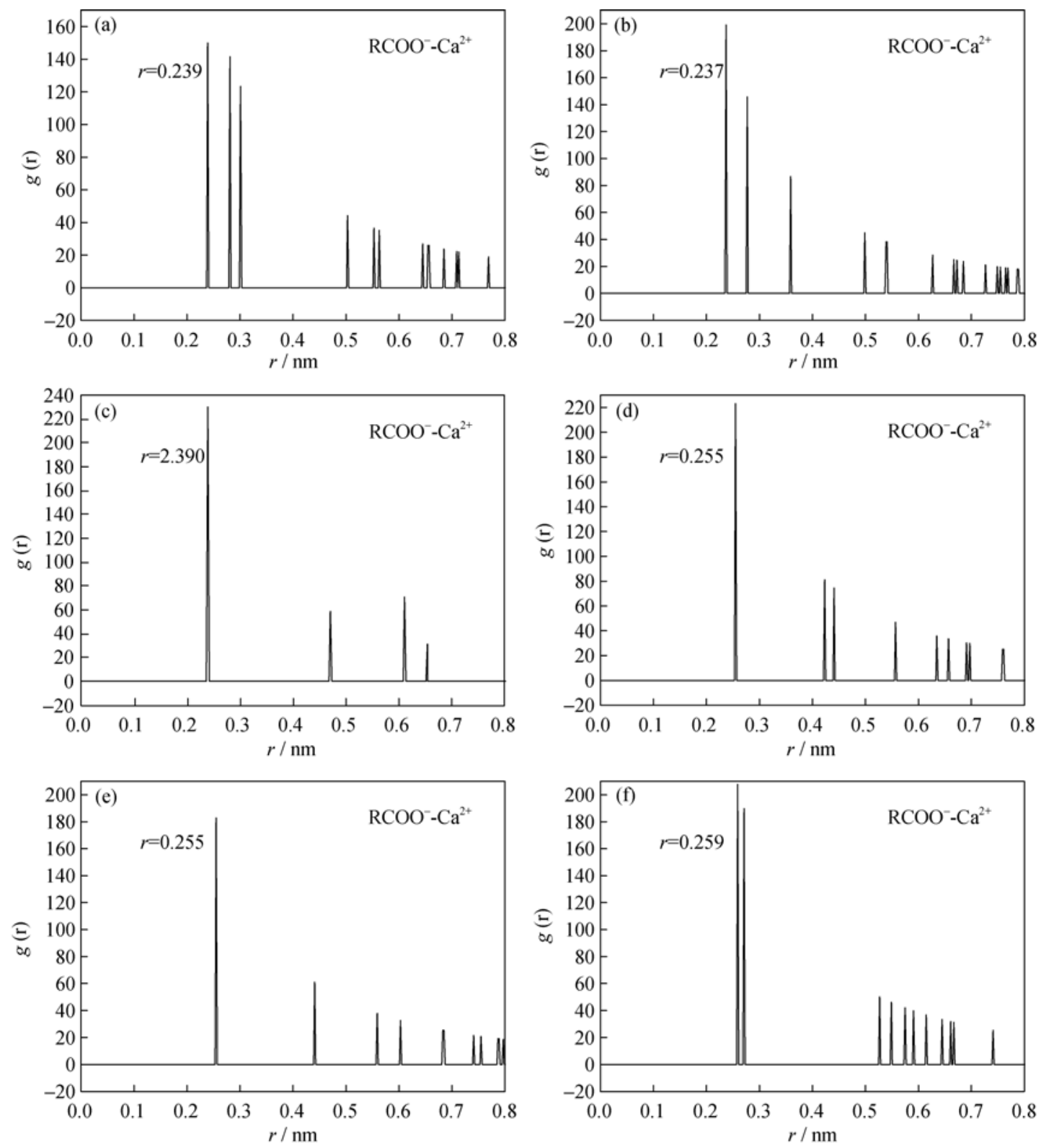

图 5 不同晶面上粒子的径向分布函数

Fig. 5 Radial distribution functions of atoms on different faces (a) (200) face; (b) (400) face; (c) (002) face; (d) (310) face; (e) (530) face; (f) (510) face

\section{参考文献:}

[1] WANG Z H, HAN Y X, YUAN Z T, et al. Calcium sulfate whiskers preparation and its application. Mining and Metallurgy, 2005, 14(2): $38-41$.

[2] WANG P, LEE E J, PARK C S, et al. Calcium sulfate hemihydrate powders with a controlled morphology for use as bone cement. Journal of the American Ceramic Society, 2008, 91(6): 2039-2042.

[3] ZHU Z C, XU L, CHEN G A. Effect of different whiskers on the physical and tribological properties of non-metallic friction materials. Materials and Design, 2011, 32(1): 54-61.

[4] LIU J Y, RENI L, WEI Q, et al. Fabrication and characterization of polycaprolactone/calcium sulfate whisker composites. Express Polymer Letters, 2011, 5(8): 742-752.

[5] WANG J C, PAN X C, XUE Y, et al. Studies on the application properties of calcium sulfate whisker in silicone rubber composites. Journal of Elastomers and Plastics, 2011, 44(1): 55-66.

[6] ROHL A L. Computer prediction of crystal morphology. Current Opinion in Solid State and Materials Science, 2003, 7(1): 21-26.

[7] GUAN B H, SHEN Z X, WU Z B, et al. Effect of $\mathrm{pH}$ on the preparation of $\alpha$-calcium sulfate hemihydrate from FGD gypsum with the hydrothermal method. Journal of the American Ceramic Society, 2008, 91(12): 3835-3840.

[8] YANG N, XIAO H N, GUO W M. Additive-assisted hydrothermal synthesis of calcium sulfate whisker its growth mechanism. Jour- 
nal of the Chinese Ceramic Society, 2014, 42(4): 539-544.

[9] JONES F, OGDEN M I. Controlling crystal growth with modifiers. CrystEngComm, 2012, 12(4): 1016-1023.

[10] GUAN B H, YANG L C, WU Z B. Effect of $\mathrm{Mg}^{2+}$ ions on the nucleation kinetics of calcium sulfate in concentrated calcium chloride solutions. Industrial \& Engineering Chemistry Research, 2010, 49(12): 5569-5574.

[11] FELDMANN T, DEMOPOULOS G P. Effects of crystal habit modifiers on the morphology of calcium sulfate dihydrate grown in strong $\mathrm{CaCl}_{2}-\mathrm{HCl}$ solutions. Journal of Chemical Technology \& Biotechnology, 2014, 89(10): 1523-1533.

[12] SCHMIDT C, ULRICH J. Morphology prediction of crystals grown in the presence of impurities and solvents-An evaluation of the state of the art. Journal of Crystal Growth, 2012, 353(1): $168-173$.

[13] ZHANG L, REN W Q, Samanta A, et al. Recent developments in computational modelling of nucleation in phase transformations. npj Computational Materials, 2016, 2(16003):1-9.

[14] YANI Y, CHOW P S, TAN R B H. Molecular simulation study of the effect of various additives on salbutamol sulfate crystal habit. Molecular Pharmaceutics, 2011, 8(5): 1910-1918.

[15] MAO X L, SONG X F, LU G M, et al. Effect of additives on the morphology of calcium sulfate hemihydrate: experimental and molecular dynamics simulation studies. Chemical Engineering Journal, 2015, 278: 320-327.

[16] YUAN Z T, WANG Y B, HAN Y X, et al. Stability of hemihydrate calcium sulfate whiskers. Chinese Journal of Inorganic Chemistry, 2008, 24(7): 1062-1067.

[17] SUN L G, HE X Q, LU J. Super square carbon nanotube network: a new promising water desalination membrane. npj Computational Materials, 2016, 2(16004): 1-7.

[18] MAYO S L, OLAFSON B D, GODDARD W A. DREIDING: A generic force field for molecular simulations. The Journal of
Physical Chemistry, 1990, 94(26): 8897-8909.

[19] KITAO O, MIURA N, USHIYAMA H. Molecular mechanics with QEq-CS (charge equilibration method generalized for charge separation system). Journal of Molecular Structure: THEOCHEM, 1999, 461-462: 239-247.

[20] WELLS B A, CHAFFEE A L. Ewald summation for molecular simulations. Journal of Chemical Theory and Computation, 2015, 11(8): 3684-3695.

[21] NI B, BAUMKETNER A. Effect of atom- and group-based truncations on biomolecules simulated with reaction-field electrostatics. Journal of Molecular Modeling, 2011, 17(11): 2883-2893.

[22] 陈正隆, 徐为人, 汤立达编著. 分子模拟的理论与实践. 北京: 化学工业出版社, 2007.

[23] FENG Q M, ZHAO Y S, ZHANG G F. Adsorption mechanisms of sodium oleate on surfaces of hematite and apatite. The Chinese Journal of Nonferrous Metals, 2012, 22(10): 2902-2907.

[24] 卫春雪. HMX/TATB 共晶机理及 TATB 晶习的理论研究. 绵阳: 西南科技大学硕士学位论文, 2010.

[25] REN X T, YANG L, ZHANG G Y, et al. Computational simulation of the crystal morphology of TATB. Chinese Journal of Explosives \& Propellants, 2010, 33(6): 43-46.

[26] PENG J H, ZHANG J X, QU J D, et al. Effect of organic acid on crystalline habit of $\alpha$-hemihydrate desulfurization gypsum and its crystal modification mechanism. Journal of the Chinese Ceramic Society, 2011, 39(10): 1711-1718.

[27] WANG L J, LIU G S, SONG X F, et al. Molecular modeling for selective adsorption of halite with dodecylmorpholine. Acta Physico-Chimica Sinica, 2009, 25(5): 963-969.

[28] Hansen J. Theory of Simple Liquids, Third Edition. Academic Press, 2006: 28-31.

[29] GAI J, GONG X, KANG W, et al. Key factors influencing water diffusion in aromatic PA membrane: hydrates, nanochannels and functional groups. Desalination, 2014, 333(1): 52-58. 